\title{
The Role of Kuwait in the Development of Early Thrombus Removal in Patients with Acute lliofemoral Vein Thrombosis: In Memory of Dr. Nael Al-Naqeeb
}

\author{
Bo Eklöf \\ Lund University, Helsingborg, Sweden
}

\author{
Key Words \\ Acute iliofemoral vein thrombosis - Early thrombus \\ removal $\cdot$ Role of Kuwait
}

\begin{abstract}
Many physicians in Kuwait have contributed to the development of the management of acute iliofemoral deep venous thrombosis utilizing open surgical thrombectomy for early thrombus removal. This concept is now accepted around the world, with new endovascular procedures replacing open surgery. Its development is described and the latest guidelines for early thrombus removal are presented.
\end{abstract}

(c) 2013 S. Karger AG, Basel

\section{Introduction: Rationale for Early Thrombus Removal}

When deep venous thrombosis (DVT) occurs, the goals of therapy are to:

- Prevent the extension or recurrence of the deep venous thrombus and fatal pulmonary embolism (PE)

- Minimize the early and late sequelae of DVT Antithrombotic therapy can accomplish the former, but it contributes little to the second goal. Particularly in iliofemoral venous thrombosis (IFVT), progressive swelling of the leg can lead to phlegmasia cerulea dolens (literally, painful blue swelling) and to increased compartmental pressure, which, in rare cases, can progress to venous gan-

\begin{tabular}{ll}
\hline KARGER & $\begin{array}{l}\text { Co 2013 S. Karger AG, Basel } \\
1011-7571 / 13 / 0232-0112 \$ 38.00 / 0 \quad \text { Karger }\end{array}$ \\
E-Mail karger@karger.com & $\begin{array}{l}\text { This is an Open Access article licensed under the terms of the } \\
\text { www.karger.com/mpp }\end{array}$ \\
& $\begin{array}{l}\text { Creative Commons Attribution-NonCommercial 3.0 Un- } \\
\text { ported license (CC BY-NC) (www.karger.com/OA-license), } \\
\text { applicable to the online version of the article only. Distribu- } \\
\text { tion permitted for non-commercial purposes only. }\end{array}$
\end{tabular}

grene and limb loss. Later, the development of severe postthrombotic syndrome (PTS) can result from persistent obstruction of the venous outflow and/or loss of valvular competence, and PE can lead to chronic pulmonary hypertension.

Most clinicians seem to focus on the initial treatment, i.e. preventing $\mathrm{PE}$, the propagation of the thrombus, or DVT recurrence, with little attention to the late sequelae, possibly swayed by the medical literature, which demon-

\section{Dedication}

Dr. Nael Al-Naqueeb died on January 30, 2004, at the age of 66 years. He was my friend and one of the finest surgeons in Kuwait. He devoted his life to improving the health care system in Kuwait, where his most valuable contribution was to establish the Oncological Surgical Unit at the Kuwait Cancer Control Center, where I had the privilege to be a part of his team on several occasions. As Hilal Al-Sayer wrote in his beautiful memorial: 'He was an exceptional person combining kindness, fun, resourcefulness, intelligence and goodness. He loved life and lived it to the full.' His dear wife Souad was recently affected by another severe loss when her son Hutheil passed away. He was a classmate in medical school with my son Hampus, who was his friend, and he was my student. It is a privilege for me to dedicate this article to Dr. Nael Al-Naqueeb.

Prof. Mohsen Yousof Al-Abdulrazzak, Dean of the Faculty of Medicine, Kuwait University, and Dr. Nael Al-Naqueeb, Undersecretary for the Ministry of Health, recruited me as professor and chairman of the Department of Surgery in 1981. With their undaunted and continued support, excellent physicians were recruited, and together we created an academic and medical system second to none in the Middle East. This progress was temporarily discontinued on August 2, 1990, due to the Iraqi invasion. 
strates in randomized clinical trials (RCTs) that antithrombotic therapy, particularly low-molecular-weight heparin, can achieve these goals. There is a lack of appreciation that the end point of these RCTs has not been the prevention of PTS. Furthermore, there is apparently also a lack of appreciation of the different natural history of IFVT compared to more distal DVT, as well as a lack of understanding of the different pathophysiologic changes that occur with time following DVT, and their relative contribution to the pathogenesis and severity of the postthrombotic sequelae.

\section{Pathophysiological Changes Associated with DVT}

The clinical outcome after DVT can be categorized into 4 basic patient subgroups: those with neither detectable obstruction nor valvular incompetence, those with obstruction alone, those with valvular incompetence alone, and those with both outflow obstruction and distal valvular incompetence. What is not well appreciated is that persisting proximal obstruction, even if it is ultimately relieved by partial recanalization and/or collateral development, can lead to progressive breakdown of the distal valves, resulting in reflux. Patients with both obstruction and valvular incompetence have severe postthrombotic sequelae, severe enough that conservative management is difficult even in compliant patients [1].

Thus, it would appear that early relief of the obstructing thrombus, by thrombolysis or thrombectomy (TE), should prevent more extensive PTS by restoring the venous outflow from the leg, as well as protecting the distal veins against progressive valvular incompetence. Another aspect of the importance of early thrombus removal is the new data appearing on the inflammatory response to DVT. A common mistake for clinicians is to treat all DVT patients with anticoagulation only, and belatedly refer those who complain of severe pain and swelling during follow-up for consideration of thrombolysis or TE - too late for them to achieve their goals.

The conclusions from the studies cited above are clear: early removal of the thrombus conveys significant benefits, and the earlier the removal, the better the outcome.

\section{Diagnosis}

Whether DVT is suspected on the basis of pain, discoloration, or swelling of one leg, or in search of a source for $\mathrm{PE}$, the diagnosis can be confirmed with accuracy via duplex scanning using color flow imaging. Clinical signs are more likely to be present in proximal DVT, i.e. IFVT, but

Early Thrombus Removal for Iliofemoral DVT in bedridden patients there may be no apparent swelling and the first signs may be those of PE (pleuritic chest pain, shortness of breath, and/or hemoptysis). Duplex scanning should interrogate not only the femoral and popliteal veins but also the iliac and calf veins; otherwise, 30\% of DVT will be missed.

Venography is mainly used now when catheter-directed interventions or surgical TE are indicated, to guide and monitor them. Standard ascending venography using foot vein injection may miss isolated iliac vein thrombosis unless an adequate contrast load is infused. If duplex scanning is not conclusive, computed tomography venography or magnetic resonance venography can be used.

\section{Indications for Intervention}

Early clot removal has clear benefits in 2 categories of patients with IFVT falling at the 2 ends of the clinical spectrum: in active healthy patients with good longevity to prevent or mitigate potentially severe late postthrombotic sequelae, and in those with massive swelling and phlegmasia cerulea dolens to mitigate early morbidity and prevent progression to venous gangrene.

Older patients with significant intercurrent disease and serious comorbidities who are unlikely to be active and live a long life, or those with distal thrombosis, should be treated conservatively. Late PTS is not likely to be an issue with them. However, even these patients, if faced with the threat of venous gangrene, may merit prompt clot removal.

The options for early removal of an acute thrombus in the proximal veins of the leg are:

- Catheter-directed thrombolysis (CDT)

- Percutaneous pharmacomechanical TE (PMT)

- Surgical TE.

\section{Treatment}

The treatment of patients with DVT has shifted to lowmolecular-weight heparin injections without monitoring on a weight-determined dosage administered mostly on an outpatient basis. The advent of new oral anticoagulants is an interesting development currently utilized. One potentially serious consequence of this trend is the practice of treating all patients with DVT in this manner, circumventing the opportunity to apply clot-removing interventions to appropriately selected patients with IFVT. 
Catheter-Directed Thrombolysis

Early trials of systemic thrombolytic therapy for proximal DVT showed that $<50 \%$ of the patients had complete lysis. The clinical benefits were frequently lost amid the significantly increased risk of major complications, predominantly bleeding [2]. Catheter-directed intrathrombus delivery of plasminogen activators has accelerated thrombus resolution, increasing the likelihood of success and diminishing bleeding complications. When delivered into the thrombus, plasminogen activators efficiently activate fibrin-bound plasminogen, thereby generating plasmin. In 1994, Semba and Dake [3] at Stanford reported their results using urokinase for CDT in 25 patients and found complete lysis in $72 \%$; in $16 / 25$ patients additional angioplasty and stenting was performed. In 2010, Baekgaard et al. [4] in Copenhagen, Denmark, reported 91\% patency without reflux in 103 patients treated with CDT using tPA for IFVT. The present CDT treatment regimen is as follows:

- Percutaneous intrathrombus infusion of tPA

- Increase in the volume of lytic infusate and decrease in the concentration of tPA

- Correction of the underlying proximal venous lesion using angioplasty and stenting

- Expectation of 80-90\% patency at 1 year with excellent clinical outcomes.

\section{Percutaneous Mechanical TE}

There are 3 devices approved by the Food and Drug Administration (FDA) in the USA today:

- AngioJet Power Pulse, which uses saline under high pressure creating a Bernoulli effect, lacing the thrombus with thrombolytic drug prior to mechanical TE

- Trellis, which combines balloon containment of the thrombus with mechanical destruction and thrombolysis

- Ekos EndoWave, which utilizes high-frequency, lowenergy ultrasound to change the thrombus structure, allowing better penetration of thrombolytic drugs

A summary of the present use of PMT is that it:

- Combines mechanical destruction with thrombolytic infusion

- Macerates, dissolves, and absorbs the thrombus

- Reduces the thrombolytic dose

- Reduces the time of infusion

- Reduces or eliminates patient time in ICU

For further information, refer to O'Sullivan's excellent review [5].

\section{Surgical TE}

The first TE for IFVT was performed by Läwen [6] in Germany in 1937. Surgery today is performed under general intubation anesthesia with $10 \mathrm{~cm}$ of post-end-expiratory pressure added during manipulation of the thrombus to prevent perioperative PE. The involved leg, the contralateral groin, and the abdomen are prepared. A cell saver is used to minimize blood loss. A longitudinal incision is made in the groin to expose the great saphenous vein, which is followed to its confluence with the common femoral vein, which is dissected up to the inguinal ligament. The superficial femoral artery is cleared off 3-4 $\mathrm{cm}$ below the femoral bifurcation for construction of the arteriovenous fistula (AVF). In primary IFVT, where the thrombus originates in the iliac vein with subsequent distal progression of the thrombus, a longitudinal venotomy is made in the common femoral vein and a venous Fogarty TE catheter is passed upward through the thrombus into the inferior vena cava (IVC). The balloon is inflated and withdrawn, with these maneuvers being repeated until no more thrombotic material can be extracted. With the balloon left inflated in the common iliac vein, a suction catheter is introduced to the level of the internal iliac vein to evacuate thrombi from this vein. Backflow is not a reliable sign of thrombus clearance since a proximal valve in the external iliac vein may be present in $25 \%$ of cases, preventing retrograde flow in a cleared vein. On the other hand, backflow can be excellent from the internal iliac vein and its tributaries despite a remaining occlusion of the common iliac vein. Therefore, an intraoperative completion venogram is mandatory. We occasionally used an angioscope, which enables the removal of residual thrombus material under direct vision, but today intravascular ultrasound is the preferred method. In early cases, the distal thrombus is usually readily extruded through the venotomy by manual massage of the leg distally, starting at the foot. The Fogarty venous catheter, with its soft flexible tip, can sometimes be advanced in retrograde fashion without significant trauma. The aim is to remove all fresh thrombi from the leg. The venotomy is closed with continuous sutures and an AVF created using the saphenous vein, anastomosing it end-to-side to the superficial femoral artery. An intraoperative venogram is performed through a catheter inserted in a branch of the AVF. After a satisfactory completion venogram, the wound is closed in layers and a closed suction drain is placed in the wound to evacuate blood and lymphatic fluid that may accumulate after the operation. 
In IFVT secondary to ascending thrombosis from the calf, the thrombus in the femoral vein may be old and adherent to the venous wall. In such cases the chance of preserving valve function has already been lost and the opportunity to restore patency is significantly diminished. A femoral segment without functioning valves will lead to distal valve dysfunction over time, much as will failure to achieve proximal patency. However, a patent iliac venous outflow plus a competent profunda collateral system will most of the time achieve normal venous function. Therefore, if iliac patency is established but the thrombus in the femoral vein is too old to be removed, it is preferable to ligate the femoral vein. If normal flow in the femoral vein cannot be reestablished, we recommend extending the incision distally and exploring the orifices of the deep femoral branches. These are isolated and venous flow is restored with a small Fogarty catheter. The femoral vein is then ligated distal to the profunda branches. In a 13-year follow-up after femoral vein ligation in this setting, Masuda et al. [7] reported excellent clinical and physiological results without PTS. Finally, if there is evidence of iliac vein obstruction on the completion venogram, which can occur in about $50 \%$ of left-sided IFVT, we recommend intraoperative endovenous iliac angioplasty and stenting.

If phlegmasia cerulea dolens was the indication considered to start the operation, fasciotomy of the calf compartments was performed in order to release the pressure and improve the circulation immediately. If there was extension of the thrombus into the IVC, the cava was approached transperitoneally through a subcostal incision. The IVC was exposed by deflecting the ascending colon and duodenum medially. Depending upon the venographic findings relative to the top of the thrombus, the IVC was controlled, usually just below the renal veins. The IVC was opened and the thrombus was removed by massage, especially of the iliac venous system. Then, if the femoral segment was involved, the operation was continued in the groin as described above. A modern alternative, a retrievable caval filter, can be introduced before the TE to protect against fatal PE and the IVC thrombus can be removed with the balloon catheter technique. Heparin should be continued for at least 5 days postoperatively and warfarin should be started on the first postoperative day and continued routinely for 6 months. The patient should be ambulated the day after the operation wearing a compression stocking and is usually discharged after a week, to return after 6 weeks for closure of the fistula.

Early Thrombus Removal for Iliofemoral DVT
The objectives of a temporary AVF are:

- To increase blood flow in the thrombectomized iliac segment

- To prevent immediate rethrombosis

- To allow time for healing of the endothelium

- To promote the development of collaterals in case of incomplete clearance or immediate rethrombosis of the iliac segment

Usually the AVF is performed between the saphenous vein and the superficial femoral artery. More distally placed AVF have not functioned in our experience.

A new percutaneous technique for fistula closure was developed by Endrys et al. [8] in Kuwait. Through a puncture of the femoral artery on the opposite, surgically untouched side, a catheter is inserted and positioned at the fistula level. Prior to release of a coil, an arteriovenogram can be performed to evaluate the patency of the iliac and caval veins, which is of prognostic value. More than $10 \%$ of patients have been shown to have remaining significant stenosis of the iliac vein despite an initially successful surgery. A percutaneous, transvenous angioplasty and stenting can be performed under the protection of the AVF, which is closed 4 weeks later.

\section{Complications and Results of Surgical TE}

One of the reasons that induced surgeons to abandon TE in the 1960s was the high mortality associated with early TE. In our series of over 200 patients, mortality was less than $1 \%$. There was no case of fatal PE in the perioperative period. In an RCT from Sweden [9], perfusion lung scans were positive on admission in $45 \%$ of all patients, with additional defects seen after 1 and 4 weeks in the conservatively treated group in 11 and $12 \%$, respectively, and in the thrombectomized group in 20 and $0 \%$, respectively. In this RCT where TE was combined with a temporary AVF, $13 \%$ had early rethrombosis of the iliac vein [10]. The iliac vein patency at 6 months was $76 \%$ in the surgical group compared to $35 \%$ in the conservative group as demonstrated by venography [10]. This significant difference was upheld after 5 and 10 years with 77 and $83 \%$ patency in the surgical group, respectively, versus 30 and $41 \%$ in the conservative group, respectively $[11,12]$. The femoropopliteal valvular competence at 6 months was $52 \%$ in the surgical group compared to $26 \%$ in the conservatively treated group, monitored by descending venography with Valsalva. This was a significant difference [10]. After 5 years, combining the results of all functional tests, $36 \%$ of the surgical patients had

Med Princ Pract 2014;23:112-118 
normal venous function compared to $11 \%$ of the conservatively treated group [11]. These differences were not statistically significant due to a loss of patients. At 10 years, using duplex scanning, popliteal reflux was found in $32 \%$ of patients in the surgical group compared to $67 \%$ in the conservative group [12].

\section{New Developments to Improve TE}

There has been significant improvement in the results of surgical TE with the understanding of the need to immediately restore iliac vein outflow in patients with iliac vein obstruction as the major cause of their DVT. Control of the iliac vein outflow immediately after TE can be achieved via an intraoperative venogram, angioscopy, or intravascular ultrasound. For the remaining obstruction, intraoperative endovenous angioplasty and stenting is recommended. Schwarzbach et al. [13] reported excellent results in 18 of 20 patients who maintained patency after 21 months of follow-up. To improve the patency and preservation of the valves in the deep veins of the leg, Blättler et al. [14] combined TE of the iliac vein with thrombolysis applied under ischemic conditions to the leg veins. None of the 33 patients experienced clinically apparent recurrence within the first year. Clinical signs of the PTS were absent in all but one patient.

With improved outflow through the iliac system by angioplasty and stenting and increased inflow from the improved distal patency by regional thrombolysis, the temporary AVF may not be necessary. An excellent review of contemporary venous TE was published by Comerota and Gale [15] in 2006.

\section{Developments in Kuwait from 1981 to 1990}

I had the privilege to work as professor and chairman of the Department of Surgery at the Faculty of Medicine of Kuwait University from 1981 to 1990. At Mubarak Al Kabeer Hospital we admitted more than 400 patients with DVT during this period, the majority with IFVT. More than 100 patients were operated with venous TE with a temporary AVF. The mean age was young, i.e. 33 years, which should be compared to the Swedish RCT where it was 65 years. The majority were women, with a high incidence before and after delivery. The tradition in Kuwait was to keep the women in bed around delivery time. In collaboration with Dipika Mohanti, Professor of Hematology, we studied the etiology in 130 patients diagnosed with DVT [16] and found fibrinolytic defects in 25\% of cases and coagulation defects in $27 \%$. The surgical technique was refined during this period. A fruitful collaboration with Jiri Endrys, a Czech interventional cardiologist at the Chest Hospital, led to the development of percutaneous closure of the AVF and postoperative angioplasty of the remaining iliac vein obstruction [8]. Six weeks after $\mathrm{TE}$, the femoral artery on the contralateral nonoperated side was catheterized and the tip of the catheter was placed at the level of the AVF. An arteriovenogram through the AVF was performed, and if the thrombectomized iliac vein was patent the AVF was closed using a detachable balloon. If there was a remaining obstruction of the iliac vein, angioplasty was performed. The landmark paper of Endrys et al. [8] was published in Cardiovascular and Interventional Radiology in 1989. This pioneering work stimulated the present approach of aggressively treating the remaining iliac vein obstruction after early thrombus removal with immediate angioplasty and stenting. A report of the results of the Kuwaiti experience of TE with AVF was published in Surgery in 1991 [17]. Patients with IFVT with an extension of the thrombus into the IVC presented a special problem. We studied 52 patients in whom we performed successful TE of the IVC through an open retroperitoneal approach in 27 patients compared to 25 patients in whom TE failed or was not attempted. At follow-up after 23 months, development of DVT in the contralateral initially not involved leg occurred in 1 patient (7\%) in whom IVC TE was successful, compared to all 25 patients $(100 \%)$ in whom IVC TE was not performed. This paper was published in the European Journal of Vascular Surgery in 1992 [18]. The development in Kuwait was also featured in 2 books: Controversies in Management of Venous Disorders in 1987 [19], where 7 chapters had authors from Kuwait, and Surgery for All in 1992 [20], with 4 chapters from Kuwait, i.e. 'Kuwait - programmed analysis of trauma', 'Vascular surgery in Kuwait', 'Variations in surgical endocrinology in the developing world', and 'The future of academic surgery in the developing world'.

\section{Current Guidelines for Early Thrombus Removal}

In a collaboration between the Society for Vascular Surgery (SVS) and the American Venous Forum, guidelines for early thrombus removal were published in the Journal of Vascular Surgery in May 2012 [21] based on the GRADE system (Grading of Recommendations, Development, and Evaluation). There are two components to any treatment recommendation (strong: 1 or weak: 2 ) based 
on the degree of confidence that the recommendation will provide more benefit than harm. The second is an evaluation of the level of evidence (A to $\mathrm{C}$ ) based on the confidence that the estimate of the effect is correct. The strength of a recommendation ( 1 or 2 ) reflects the balance of benefits and risks as well as the cost to the health care system. In GRADE language, 'recommending' means the use of strong GRADE 1 guidelines, while 'suggesting' means the use of weaker GRADE 2 guidelines.

\section{Precision in the Diagnosis}

We recommend the use of precise anatomic terminology to characterize the most proximal extent of DVT involving:

- Iliofemoral veins with or without extension into the IVC

- Femoropopliteal veins

- Veins isolated to the calf

contrary to simple characterization of a thrombus as proximal or distal - $1 \mathrm{~A}$.

\section{Diagnosis}

If iliofemoral DVT is suspected but not confirmed by duplex scanning, we recommend:

- Computed tomography venography

- Magnetic resonance venography

to characterize the most proximal thrombus extent - 1C.

\section{Indications for Early Thrombus Removal}

We suggest a strategy of early thrombus removal in selected patients meeting the following criteria:

- A first episode of acute iliofemoral DVT

- Symptoms $<14$ days in duration

- A low risk of bleeding

- Ambulatory with good functional capacity and an acceptable life expectancy - 2C

We recommend early thrombus removal as the treatment of choice in patients with limb-threatening venous ischemia due to iliofemoral DVT with or without associated femoropopliteal venous thrombosis, i.e. phlegmasia cerulea dolens - $1 \mathrm{~A}$.

We recommend that patients with isolated femoropopliteal DVT be managed with conventional anticoagulation therapy because there is currently insufficient evidence to support early thrombus removal strategies in this patient population.

\section{Techniques for Early Thrombus Removal}

We suggest percutaneous catheter-based techniques (CDT or PMT) as first-line therapy $-2 \mathrm{C}$.

Early Thrombus Removal for Iliofemoral DVT
We suggest a strategy of PMT be considered over CDT alone if expertise and resources are available $-2 \mathrm{C}$.

We suggest open surgical TE in selected patients who are candidates for anticoagulation but in whom thrombolytic therapy is contraindicated $-2 \mathrm{C}$.

\section{IVC Filters}

We recommend against the routine use of IVC filters (permanent of temporary) in conjunction with CDT of the iliofemoral venous segments - $1 \mathrm{C}$.

We suggest that the relative risks versus the benefits of periprocedural retrievable IVC filter placement be considered in patients undergoing PMT and in those with a thrombus extending into the IVC or having markedly limited cardiopulmonary reserve $-2 \mathrm{C}$.

\section{Adjunctive Use of Venous Stents}

We recommend the use of self-expanding metallic stents for the treatment of chronic iliocaval compressive or obstructive lesions that are uncovered by any of the thrombus removal strategies - $1 \mathrm{C}$.

We suggest that stents not be used in the femoral and popliteal veins $-2 \mathrm{C}$.

\section{Conventional Treatment}

We recommend that patients managed with early thrombus removal be treated with a standard course of conventional anticoagulation after the procedure $-1 \mathrm{~A}$.

We recommend that all patients be treated with kneehigh compression stockings (30-40 $\mathrm{mm} \mathrm{Hg}$ ) for at least 2 years after the procedure $-1 \mathrm{C}$.

\section{Summary and Recommendations}

Early thrombus removal is recommended in the new guidelines from the SVS/American Venous Forum in the USA. There are 3 ongoing RCTs:

- CaVenT in Norway, testing CDT versus conventional anticoagulation. Two-year follow-up is published showing significantly less PTS after CDT [22].

- ATTRACT in the USA, testing CDT/PMT versus conventional anticoagulation. This is a multicenter study that will include 692 patients, and the inclusion rate is slow. Currently, over 480 patients are included.

- CAVA in The Netherlands, testing ultrasound-assisted thrombolysis versus conventional anticoagulation. The inclusion rate is slow.

Kuwait has played a role in the development of early thrombus removal through: 
- Development of the surgical technique

- Development of percutaneous closure of the AVF

- Development of percutaneous balloon dilatation of the remaining iliac vein obstruction

- Proving the importance of removing IVC thrombosis to avoid contralateral DVT

The future challenge is to follow up the long-term results of patients in Kuwait after 20-30 years comparing patients who underwent TE + AVF with those who were treated conservatively.

\section{Conclusion}

Kuwait has played a role in the development of early thrombus removal through its development of the surgical technique, percutaneous closure of the AVF, and percutaneous balloon dilatation of any remaining iliac vein obstruction. It has proven the importance of removing IVC thrombosis to avoid contralateral DVT. Future challenges are to follow up the long-term results of patients in Kuwait after 20-30 years, comparing patients who underwent TE + AVF with those who were treated conservatively.

\section{References}

1 Eklöf B, Rutherford RB: Surgical thrombectomy for acute deep venous thrombosis; in Rutherford RB (ed): Vascular Surgery, ed 6. Philadelphia, Elsevier Saunders, 2005, pp 2188-2197.

2 Jacobsen B: Thrombolytic treatment in venous thromboembolism; in Eklöf B, Gjöres JE, Thulesius O, et al (eds): Controversies in the Management of Venous Disorder. London, Butterworths, 1989, pp 116-126.

3 Semba CP, Dake MD: Iliofemoral deep vein thrombosis: aggressive therapy with catheterdirected thrombolysis. Radiology 1994;191: 487-494.

4 Baekgaard N, Broholm R, Just S, et al: Longterm results using catheter-directed thrombolysis in 103 lower limbs with acute iliofemoral venous thrombosis. Eur J Vasc Endovasc Surg 2010;39:112-117.

5 O'Sullivan GJ: The role of interventional radiology in the management of deep venous thrombosis: advanced therapy. Cardiovasc Intervent Radiol 2011;34:445-461.

6 Läwen A: Über Thrombektomie bei Venenthrombose und Arteriospasmus. Zentralbl Chir 1937;64:961-968.

7 Masuda EM, Kistner RL, Ferris EB: Longterm effects of superficial femoral vein ligation: thirteen year follow-up. J Vasc Surg 1992;16:741-749.
8 Endrys J, Eklöf B, Neglén P, et al: Percutaneous balloon occlusion of surgical arteriovenous fistulae following venous thrombectomy. Cardiovasc Intervent Radiol 1989;12: 226-229.

-9 Plate G, Ohlin P, Eklöf B: Pulmonary embolism in acute iliofemoral venous thrombosis. Br J Surg 1985;72:912-915.

10 Plate G, Einarsson E, Ohlin P, et al: Thrombectomy with temporary arteriovenous fistula: the treatment of choice in acute iliofemoral venous thrombosis. J Vasc Surg 1984;1:867876.

11 Plate G, Åkesson H, Einarsson E, et al: Longterm results of venous thrombectomy combined with temporary arteriovenous fistula. Eur J Vasc Surg 1990;4:483-489.

12 Plate G, Eklöf B, Norgren L, et al: Venous thrombectomy for iliofemoral vein thrombosis: 10-year results of a prospective randomized study. Eur J Vasc Endovasc Surg 1997;14: 367-374.

13 Schwarzbach MH, Schumacher H, Böckler D, et al: Surgical thrombectomy followed by intraoperative endovascular reconstruction for symptomatic iliofemoral venous thrombosis. Eur J Vasc Endovasc Surg 2005;29:58-66.

14 Blättler W, Heller G, Largiadèr J, et al: Combined regional thrombolysis and surgical thrombectomy for treatment of iliofemoral vein thrombosis. J Vasc Surg 2004;40:620625.
15 Comerota AJ, Gale SS: Technique of contemporary iliofemoral and infrainguinal venous thrombectomy. J Vasc Surg 2006;43:185-191.

16 Mohanti D, Das KC, Al-Hussein H, et al Thrombophilia in ethnic Arabs in Kuwait. Ann Hematol 1996;73:283-290.

17 Neglén P, Al-Hassan H, Endrys J, et al: Iliofemoral venous thrombectomy followed by percutaneous closure of a temporary arteriovenous fistula. Surgery 1991;110:493-499.

18 Neglén P, Nazzal M, Al-Hassan H, et al: Surgical removal of an inferior vena cava thrombus. Eur J Vasc Surg 1992;6:78-82.

19 Eklöf B, Gjöres JE, Thulesius O, et al (eds): Controversies in the Management of Venous Disorders. London, Butterworths, 1989.

20 Ahmed M, Blanchard RJW, Eklöf B, et al (eds): Surgery for All: A View from the Developing World. Lahore, Ferozsons, 1992.

21 Meissner MH, Gloviczki P, Comerota AJ, et al: Early thrombus removal strategies for acute deep venous thrombosis: clinical practice guidelines of the Society for Vascular Surgery and the American Venous Forum. J Vasc Surg 2012;55:1449-1462.

22 Enden T, Haig Y, Klöw NE, et al: Long-term outcome after additional catheter-directed thrombolysis versus standard treatment for acute iliofemoral deep vein thrombosis (the CaVenT study): a randomised controlled study. Lancet 2012;379:31-38. 